\title{
Vitamin $D$ and disease severity in coronavirus disease 19 (COVID-19)
}

\author{
G. Adami ${ }^{1 *}$, A. Giollo ${ }^{1 *}$, A. Fassio ${ }^{1}$, C. Benini ${ }^{1}$, E. Bertoldo ${ }^{1}$, F. Bertoldo ${ }^{2}$, \\ G. Orsolini', L. Idolazzi ${ }^{1}$, O. Viapiana ${ }^{1}$, S. Giannini ${ }^{3}$, G. Passeri', E. Tacconelli, \\ C. Micheletto ${ }^{6}$, D. Gatti' ${ }^{1}$, M. Rossini ${ }^{1}$ \\ ${ }^{1}$ Rheumatology Unit, University of Verona, Italy; ${ }^{2}$ Internal Medicine Unit, University of Verona, Italy; \\ ${ }^{3}$ Internal Medicine Unit, University of Padua, Italy; ${ }^{4}$ Internal Medicine Unit, University of Parma, Italy; \\ ${ }^{5}$ Infectious Diseases Unit, University of Verona, Italy; ${ }^{6}$ Pulmonary Unit, University of Verona, Italy \\ ${ }^{*}$ These two authors contributed equally to this work
}

\begin{abstract}
SUMMARY
The role of 25-OH-vitamin D in the assessment of coronavirus disease 19 (COVID-19) has not been investigated. We sought to investigate the prevalence of 25-OH-vitamin D deficiency among COVID-19 patients, and to determine the associations between $25-\mathrm{OH}$-vitamin $\mathrm{D}$ status and the severity of the disease.

We have conducted a retrospective observational study of COVID-19 patients admitted to the University of Verona Hospital Trust. Demographic, clinical and biochemical parameters were collected at hospital admission, and serum 25-OH-vitamin D levels were measured. The following outcomes were assessed: arterial partial oxygen pressure $\left(\mathrm{PaO}_{2}\right)$; C-reactive protein (CRP); length of hospitalization; requirement of oxygen therapy; non-invasive ventilation (NIV); mechanical ventilation; and death.

Among 61 patients enrolled, $72.1 \%$ was $25-\mathrm{OH}$-vitamin D deficient $(<20 \mathrm{ng} / \mathrm{mL})$ and $57.4 \%$ had $25-\mathrm{OH}-$ vitamin $\mathrm{D}<15 \mathrm{ng} / \mathrm{mL}$. Patients with arterial $\mathrm{PaO}_{2}<60 \mathrm{mmHg}$ had significantly lower mean 25-OH-vitamin D levels compared to patients with $\mathrm{PaO}_{2} \geq 60 \mathrm{mmHg}$ ( $13.3 \mathrm{ng} / \mathrm{mL}$ vs $20.4 \mathrm{ng} / \mathrm{mL}$ respectively, $\left.\mathrm{p}=0.03\right)$. Vitamin $\mathrm{D}$ deficiency was associated with 3 -fold higher risk of having arterial $\mathrm{pO}_{2}<60 \mathrm{mmHg}$. 25-OH-vitamin D deficiency was associated with increased CRP and dyspnea.

25-OH-vitamin D deficiency was associated with more severe systemic inflammatory response and respiratory failure in COVID-19 patients.
\end{abstract}

Key words: COVID-19; vitamin D; SARS-CoV-2.

\section{INTRODUCTION}

he coronavirus disease 19 (COV-

ID-19), a novel acute respiratory disease caused by the severe acute respiratory distress syndrome coronavirus 2 (SARSCoV-2), has received worldwide attention. The clinical spectrum of COVID-19 ranges from asymptomatic infection to life-threatening disease due to acute respiratory distress syndrome (ARDS). In Italy, as of May 9, more than 200,000 cases had been recorded and up to $16 \%$ of the patients requiring hospitalization were admitted to intensive care units (ICUs) (1). SARS$\mathrm{CoV}-2$ is a $\beta$-coronavirus that causes interstitial pneumoniae by attaching to angiotensin-converting enzyme 2 (ACE2) recep- tors in the respiratory tract (2). There is growing evidence that the immune system and its response to SARS-CoV-2 infection plays a crucial role in the development of severe complications of the disease. Indeed, it has been increasingly recognized that the ARDS occurring in many severe cases of COVID-19 is due to an uncontrolled inflammatory response to the virus, which involves the production of high levels of pro-inflammatory cytokines such as interleukin-1 and interleukin-6 $(3,4)$.

Among the factors that influence our immune system, vitamin D and its metabolite 25-OH-vitamin D are known to have pleiotropic and multiple effects (5). Vitamin D deficiency (i.e., 25-OH-vitamin D <20 ng/ $\mathrm{mL}$ ) is common among people of all ages $\overline{\text { Corresponding author: }}$ Giovanni Adami Rheumatology Unit, Department of Medicine University of Verona, Policlinico G.B. Rossi, Verona, Italy E-mail: adami.g@yahoo.com 
all over Europe (6). Low 25-OH-vitamin D serum levels have been associated with increased production of pro-inflammatory cytokines, such as IL-6, and with more severe clinical manifestations in patients with pneumonia (e.g., development of ARDS) (7). By acting on different pathways, vitamin D can modulate both the innate and adaptive systems thanks to its ubiquitously distributed receptors. Of particular interest is the hypothesized dual action of vitamin $\mathrm{D}$ on the mucosal defenses, indeed, vitamin $\mathrm{D}$ receptor (VDR) has been found on both resident immune cells and respiratory epithelial cells (8).

The aim of the present study is to describe the vitamin D status among hospitalized COVID-19 patients, and to define the relationship between 25-OH-D serum levels at hospital admission and COVID-19 disease severity.

\section{MATERIALS AND METHODS}

We conducted a retrospective analysis on patients admitted to the University of Verona hospital for COVID-19. We collected demographic, clinical, biochemical and radiological characteristics, as well as treatment and outcome data, from electronic medical records. We obtained complete medical histories, including underlying chronic diseases, symptoms (including dyspnea defined as difficult breathing or shortness of breath), clinical signs, laboratory findings, computed tomographic scans of the chest and/or standard radiography, and treatment (including antiviral therapy, antibiotics, corticosteroid therapy, and oxygen support) administered during the hospital admission. All the patients were tested at admission for serum 25-OH-vitamin D (LIAISON $^{\circledR} 25 \mathrm{OH}$ Vitamin D assay, DiaSorin, Italy, a direct competitive chemiluminescent immunoassay; the intra-assay variation coefficient was $8 \%$ and the interassay variation coefficient was $12 \%$ ), blood gas analysis, complete blood count, liver and kidney function, creatine kinase, lactate dehydrogenase, ferritin, fibrinogen, Creactive protein (CRP), procalcitonin (PCT) and d-dimer.
Continuous variables are presented as mean and standard deviation if normally distributed or median and interquartile range if they are not. Group comparisons were performed with $t$-student and MannWhitney $U$ tests (for normally and nonnormally distributed continuous variables, respectively). Associations between continuous variables were tested using Pearson correlation coefficients and multivariate linear regression. We compared proportions for categorical variables by using the $\chi^{2}$ test. We used Fisher's exact test in the analysis of contingency tables when the sample sizes were small. All differences were considered significant when the $p$ value was inferior or equal to 0.05 . All analyses were performed with SPSS Version 26 (SPSS, Inc., Chicago, IL, USA). All patients provided informed consent and access to all clinical data was allowed as per study protocol (2598CESC), which was approved by the local ethics committee. All the analyses have been conducted in full accordance with the ethical standards of the qualified institutional and national committees on human subjects as well as with the Helsinki Declaration.

\section{RESULTS}

We consecutively enrolled 61 patients between March $8^{\text {th }}$ and May $8^{\text {th }}, 2020$. Clinical and laboratory characteristics were collected for the whole sample. The mean age was 69.4 years with no gender differences in the study sample. Tables I and II show the demographic, clinical and laboratory characteristics of the population under analysis. We found a large prevalence of vitamin D deficiency, irrespective of the threshold adopted, with approximately $70 \%$ of the population having less than 20 $\mathrm{ng} / \mathrm{mL}$ (Figure 1). Of note, the subjects younger than 50 years of age and 60 years of age ( $n=8$ and $n=19$ respectively) had mean vitamin $D$ serum levels of $9.5 \mathrm{ng} / \mathrm{mL}$ and $11.9 \mathrm{ng} / \mathrm{mL}$ respectively. We did not find any significant correlation between vitamin D serum levels and age or gender. We found that patients with $\mathrm{pO}_{2}<60 \mathrm{mmHg}$ had significantly lower levels of serum 
25-OH-vitamin $\mathrm{D}$ compared to patients with $\mathrm{pO}_{2} \geq 60 \mathrm{mmHg}(13.3 \mathrm{ng} / \mathrm{mL}$ vs 20.4 $\mathrm{ng} / \mathrm{mL}$ respectively, $\mathrm{p}=0.03$ ) (Figure 2). Among the 44 patients with vitamin $\mathrm{D}$ deficiency ( $<20 \mathrm{ng} / \mathrm{mL}$, average $13.3 \mathrm{ng} / \mathrm{mL}$ ), $56.8 \%$ had $\mathrm{pO} 2$ levels on admission lower than $60 \mathrm{mmHg}$ whilst this was found in only $29.4 \%$ of patients with vitamin $\mathrm{D} \geq 20$ $\mathrm{ng} / \mathrm{mL}$ (average $20.4 \mathrm{ng} / \mathrm{mL})\left(\chi^{2} 3.68\right.$, $\mathrm{p}=0.055)$. Patients with vitamin $\mathrm{D}$ serum $<20 \mathrm{ng} / \mathrm{mL}$ were more prone to be dyspneic compared to non-deficient patients. Among dyspneic patients, $56.8 \%$ had vitamin $\mathrm{D}$ deficiency, this proportion was mar-

Table I - Clinical characteristics of the study population divided by vitamin D serum levels.

\begin{tabular}{|c|c|c|c|c|}
\hline Characteristics & $\begin{array}{c}\text { Complete cohort } \\
n=61\end{array}$ & $\begin{array}{c}25-\mathrm{OH} \text {-vitamin } \mathrm{D}< \\
20 \mathrm{ng} / \mathrm{mL} \mathrm{n}=44\end{array}$ & $\begin{array}{l}25-0 \mathrm{OH} \text {-vitamin D } \\
\geq 20 \mathrm{ng} / \mathrm{mL} \mathrm{n}=17\end{array}$ & $p$ value \\
\hline \multicolumn{5}{|l|}{ Demographic characteristics } \\
\hline Age, years (SD) & $69.4(15.3)$ & $67.7(16.2)$ & $74.0(12.0)$ & NS \\
\hline Sex, male (\%) & $32(52.5)$ & $25(56.8)$ & $7(41.2)$ & NS \\
\hline Weight, kg (SD) & $73.1(15.1)$ & $73.4(16.4)$ & $72.4(13.3)$ & NS \\
\hline Height, cm (SD) & $167(9)$ & $168(8)$ & $162(9)$ & NS \\
\hline \multicolumn{5}{|l|}{ Clinical characteristics on admission } \\
\hline Time to first symptom, days (IQR) & $5(2.5-8-5)$ & $6.5(0.8-8.5)$ & $5(3.0-9.0)$ & NS \\
\hline Temperature, ${ }^{\circ} \mathrm{C}(\mathrm{IQR})$ & $37.9(36.4-38.3)$ & $37.7(36.4-38.3)$ & $38.0(36.8-38.5)$ & NS \\
\hline $\begin{array}{l}\text { Distribution of temperature (\%) } \\
<37.5^{\circ} \mathrm{C} \\
37.5-38.0^{\circ} \mathrm{C} \\
38.1-39.0^{\circ} \mathrm{C} \\
>39^{\circ} \mathrm{C}\end{array}$ & $\begin{array}{c}24(45.3) \\
11(20.8) \\
15(28.3) \\
3(5.7) \\
\end{array}$ & $\begin{array}{l}18(47.4) \\
8(21.1) \\
10(26.3) \\
2(5.3) \\
\end{array}$ & $\begin{array}{l}6(40.0) \\
3(20.0) \\
5(33.3) \\
1(6.7) \\
\end{array}$ & NS \\
\hline Systolic blood pressure, mmHg (SD) & $129(26)$ & $131(28)$ & $122(22)$ & NS \\
\hline Diastolic blood pressure, $\mathrm{mmHg}(\mathrm{SD})$ & $74(12)$ & $75(13)$ & $71(11)$ & NS \\
\hline Heart rate, bpm (SD) & $86(20)$ & $86(21)$ & $87(12)$ & NS \\
\hline Respiratory rate (SD) & $23(5)$ & $23(5)$ & $22(4)$ & NS \\
\hline Fever (Temperature $\left.>37.5^{\circ} \mathrm{C}\right)(\%)$ & $43(70.5)$ & $30(68.2)$ & $13(76.5)$ & NS \\
\hline Diarrhea (\%) & $6(9.8)$ & $4(9.1)$ & $2(11.8)$ & NS \\
\hline Sore throat (\%) & $2(3.3)$ & $2(4.5)$ & $0(0)$ & NS \\
\hline Anosmia or ageusia (\%) & $3(4.9)$ & $3(6.8)$ & $0(0)$ & NS \\
\hline Cough (\%) & $23(37.7)$ & $18(40.9)$ & $5(29.4)$ & NS \\
\hline Sputum production (\%) & $2(3.3)$ & $1(2.3)$ & $1(5.9)$ & NS \\
\hline Dyspnea (\%) & $30(49.9)$ & $25(56.8)$ & $5(29.4)$ & 0.055 \\
\hline Interstitial pneumonia on chest X-ray / HRCT (\%) & $52(86.7)$ & $39(88.6)$ & $13(76.5)$ & NS \\
\hline \multicolumn{5}{|l|}{ Treatment during hospitalization } \\
\hline Hydroxychloroquine (\%) & $40(65.6)$ & $32(72.7)$ & $15(88.2)$ & NS \\
\hline Tocilizumab (\%) & $9(14.8)$ & $9(20.5)$ & $0(0)$ & 0.04 \\
\hline Lopinavir / Ritonavir (\%) & $40(65.6)$ & $28(63.6)$ & $12(70.6)$ & NS \\
\hline Intravenous methylprednisolone (\%) & $20(32.8)$ & $14(31.8)$ & $6(35.3)$ & NS \\
\hline $\begin{array}{l}\text { Antibiotics (\%) } \\
\text { None } \\
\text { Ceftriaxone } \\
\text { Piperacillin/Tazobactam } \\
\text { Azithromycin } \\
\text { Others }\end{array}$ & $\begin{array}{l}19(31.1) \\
21(34.4) \\
10(16.4) \\
10(16.4) \\
1(1.6)\end{array}$ & $\begin{array}{l}11(25.0) \\
18(40.9) \\
7(15.9) \\
7(15.9) \\
1(2.3)\end{array}$ & $\begin{array}{c}8(47.1) \\
3(17.6) \\
3(17.6) \\
3(17.6) \\
0(0)\end{array}$ & NS \\
\hline
\end{tabular}


Continue $\gg>$

\begin{tabular}{|c|c|c|c|c|}
\hline Characteristics & $\begin{array}{c}\text { Complete cohort } \\
n=61\end{array}$ & $\begin{array}{c}25-O H \text {-vitamin D }< \\
20 \mathrm{ng} / \mathrm{mL} \mathrm{n}=44\end{array}$ & $\begin{array}{l}\text { 25-OH-vitamin D } \\
\geq 20 \mathrm{ng} / \mathrm{mL} \mathrm{n}=17\end{array}$ & $\mathrm{p}$ value \\
\hline \multicolumn{5}{|l|}{ Demographic characteristics } \\
\hline $\begin{array}{l}\text { Cholecalciferol (\%) } \\
\text { None } \\
<1,000 \text { IU/day } \\
\geq 1,000 \text { IU/day }\end{array}$ & $\begin{array}{c}54(88.5) \\
1(1.6) \\
6(9.9)\end{array}$ & $\begin{array}{c}44(100) \\
0(0) \\
0(0)\end{array}$ & $\begin{array}{c}10(58.8) \\
1(5.9) \\
6(35.3)\end{array}$ & 0.001 \\
\hline \multicolumn{5}{|l|}{ Outcomes during hospitalization } \\
\hline Hospital stay length, days $(\mathrm{IQR})^{\star}$ & $17.0(7.0-26.5)$ & $17(7.0-29.0)$ & $14(5.0-22.0)$ & NS \\
\hline $\begin{array}{l}\text { Required oxygen therapy (\%) } \\
\text { None } \\
\text { Low-flow nasal cannulas } \\
\text { High-flow nasal cannulas } \\
\text { Venturi mask } \\
\text { Non-rebreather mask }\end{array}$ & $\begin{array}{c}26(42.6) \\
9(14.8) \\
3(4.9) \\
10(16.4) \\
12(19.7)\end{array}$ & $\begin{array}{c}17(38.6) \\
6(13.6) \\
2(4.5) \\
9(20.5) \\
9(20.5)\end{array}$ & $\begin{array}{c}9(52.9) \\
3(17.6) \\
1(5.9) \\
1(5.9) \\
3(17.6)\end{array}$ & NS \\
\hline Required non-invasive ventilation (\%) ${ }^{*}$ & $19(55.9 \%$ of 34$)$ & $15(78.9 \%)$ & $4(21.0 \%)$ & NS \\
\hline Required mechanical ventilation (\%) ${ }^{*}$ & $8(23.5 \%$ of 34$)$ & $2(33.3 \%)$ & $6(66.6 \%)$ & NS \\
\hline Death $(\%)^{*}$ & $5(14.7 \%$ of 34$)$ & $2(40.0 \%)$ & $3(60.0 \%)$ & NS \\
\hline \multicolumn{5}{|l|}{ Coexisting comorbidities } \\
\hline $\begin{array}{l}\text { Smoking history (\%) } \\
\text { Never } \\
\text { Former } \\
\text { Current }\end{array}$ & $\begin{array}{c}40(78.4) \\
1(2.0) \\
10(19.6)\end{array}$ & $\begin{array}{c}28(63.6) \\
1(2.3) \\
7(15.9)\end{array}$ & $\begin{array}{c}12(70.6) \\
0(0) \\
3(17.6)\end{array}$ & $\begin{array}{l}\text { NS } \\
\text { NS } \\
\text { NS }\end{array}$ \\
\hline Cardiovascular diseases (\%) & $17(27.8)$ & $12(27.3)$ & $5(29.4)$ & NS \\
\hline Type 2 diabetes (\%) & $11(18.0)$ & $9(20.5)$ & $2(11.8)$ & NS \\
\hline Hypertension (\%) & $36(59.0)$ & $27(61.4)$ & $9(52.9)$ & NS \\
\hline Cancer (\%) & $11(18.0)$ & $9(20.5)$ & $2(11.8)$ & NS \\
\hline Chronic kidney disease (\%) & $11(18.0)$ & $9(20.5)$ & $2(11.8)$ & NS \\
\hline Chronic obstructive pulmonary disease (\%) & $7(11.5)$ & $5(11.4)$ & $2(11.8)$ & NS \\
\hline
\end{tabular}

${ }^{*}$ Data available in 34 patients.

ginally higher compared to $29.4 \%$ of patients with normal vitamin $\mathrm{D}$ serum levels $\left(\chi^{2} 3.68, \mathrm{p}=0.055\right)$.

We found a significant relationship between vitamin D status $(<15 \mathrm{ng} / \mathrm{mL} v s \geq 15$ $\mathrm{ng} / \mathrm{mL})$ and CRP levels $(\leq 10 \mathrm{mg} / \mathrm{L} v s>10$ $\mathrm{mg} / \mathrm{L}$ ). Indeed, patients with $25-\mathrm{OH}$-vitamin D below $15 \mathrm{ng} / \mathrm{mL}$ were more likely to show increased levels of CRP on admission $\left(\chi^{2} 4.78, p=0.02\right)$. We also found that patients with 25-OH-vitamin D below $20 \mathrm{ng} /$ $\mathrm{mL}$ had a 3-fold higher risk of having CRP above $50 \mathrm{mg} / \mathrm{L}(\mathrm{n}=28,63.8 \%)$ compared to patients with normal vitamin $\mathrm{D} \quad(\mathrm{n}=6$, $35.3 \%), \chi^{2} 3.99, p=0.04$.

During hospitalization, vitamin D deficient patients were treated more frequently with tocilizumab when compared with non-deficient individuals $(20.5 \%$ vs $0 \% \mathrm{p}=0.04)$.
We found no association between vitamin $\mathrm{D}$ deficiency and other medications.

Not surprisingly, arterial $\mathrm{pO}_{2}$ was strongly associated with duration of hospitalization, risk of non-invasive ventilation (NIV) and mechanical ventilation. We found that patients with arterial $\mathrm{pO}_{2}<60 \mathrm{mmHg}$ had a 10-times higher risk of receiving NIV (OR $10.3,95 \%$ CI 2.5-41.7, p<0.0001) and approximately 4-times higher risk of being mechanically ventilated during the hospitalization (OR 3.8, 95\% CI 1.1-13.9, $\mathrm{p}=0.04$ ).

Among other laboratory markers tested on admission, we found that higher fibrinogen and higher CRP were associated with lower $\mathrm{pO}_{2}$ on blood gas analysis. Fibrinogen levels were $6.3 \mathrm{~g} / \mathrm{L}$ and $5.3 \mathrm{~g} / \mathrm{L}$ for $\mathrm{pO}_{2}$ lower than $60 \mathrm{mmHg}$ and $\mathrm{pO}_{2}$ greater than 
Table II - Biochemical characteristics of the study population.

\begin{tabular}{|c|c|c|c|c|}
\hline \multicolumn{5}{|l|}{ Biochemical parameter } \\
\hline Distribution of laboratory findings & All cohort $n=61$ & $\begin{array}{l}25-\mathrm{OH} \text {-vitamin D } \\
<20 \mathrm{ng} / \mathrm{mL} \mathrm{n}=44\end{array}$ & $\begin{array}{l}25-\mathrm{OH} \text {-vitamin D } \\
\geq 20 \mathrm{ng} / \mathrm{mL} \mathrm{n}=17\end{array}$ & $\mathrm{OR}(95 \% \mathrm{Cl})$ \\
\hline $\begin{array}{l}25-\mathrm{OH} \text {-vitamin } \mathrm{D}, \mathrm{ng} / \mathrm{mL}<20 \mathrm{ng} / \mathrm{mL} \\
25-\mathrm{OH} \text {-vitamin } \mathrm{D}, \mathrm{ng} / \mathrm{mL}<15 \mathrm{ng} / \mathrm{mL} \\
25-\mathrm{OH} \text {-vitamin } \mathrm{D}, \mathrm{ng} / \mathrm{mL}<10 \mathrm{ng} / \mathrm{mL} \\
25-\mathrm{OH} \text {-vitamin D, ng/mL }<5 \mathrm{ng} / \mathrm{mL}\end{array}$ & $\begin{array}{l}44(72.1) \\
35(57.4) \\
19(31.1) \\
8(13.1)\end{array}$ & NA & NA & NA \\
\hline C-reactive protein $>50 \mathrm{mg} / \mathrm{L}(\%)$ & $34(55.7)$ & $28(63.6)$ & $6(35.3)$ & $3.2(1.0-10.3)$ \\
\hline Procalcitonin $>0.5 \mathrm{ng} / \mathrm{mL}(\%)$ & $11(18.0)$ & $7(15.9)$ & $4(23.5)$ & $0.6(0.1-2.4)$ \\
\hline Hemoglobin $<10 \mathrm{~g} / \mathrm{dL}(\%)$ & $6(9.8)$ & $5(11.4)$ & $1(5.9)$ & $2.0(0.2-18.9)$ \\
\hline Platelets $<150.000 / \mathrm{mm}^{3}(\%)$ & $8(13.1)$ & $7(15.9)$ & $1(5.9)$ & $3.0(0.3-26.6)$ \\
\hline Leukocytes $<4,000 / \mathrm{mm}^{3}(\%)$ & $8(13.1)$ & $6(13.5)$ & $2(11.8)$ & $2.0(0.2-17.5)$ \\
\hline Lymphocytes $<1,200 / \mathrm{mm}^{3}(\%)$ & $38(62.3)$ & $29(65.9)$ & $9(52.9)$ & $1.7(0.5-5.3)$ \\
\hline Neutrophils $<1,800 / \mathrm{mm}^{3}(\%)$ & $5(8.2)$ & $3(6.8)$ & $2(11.8)$ & $0.5(0.1-3.6)$ \\
\hline $\begin{array}{l}\text { Fibrinogen }>4.0 \mathrm{~g} / \mathrm{L}(\%) \\
\text { Fibrinogen }>5.5 \mathrm{~g} / \mathrm{L}(\%)\end{array}$ & $\begin{array}{l}46(75.4) \\
34(55.7)\end{array}$ & $\begin{array}{l}33(75.0) \\
25(56.8)\end{array}$ & $\begin{array}{l}13(76.5) \\
9(52.9)\end{array}$ & $\begin{array}{l}0.9(0.2-3.4) \\
1.2(0.4-3.6)\end{array}$ \\
\hline D-dimer $>500$ ug $/ \mathrm{L}(\%)$ & $50(82.0)$ & $37(84.1)$ & $13(76.5)$ & $1.6(0.4-6.5)$ \\
\hline Ferritin $>300$ ug/L (\%) & $48(78.7)$ & $37(84.1)$ & $11(64.7)$ & $2.9(0.8-10.4)$ \\
\hline Lactate dehydrogenase $>250$ IU/L (\%) & $42(68.9)$ & $31(70.5)$ & $11(64.7)$ & $1.3(0.4-4.2)$ \\
\hline Creatinine phosphokinase >200 IU/L (\%) & $11(18.0)$ & $9(20.5)$ & $2(11.8)$ & $1.9(0.4-10.0)$ \\
\hline Creatinine $>1.0 \mathrm{mg} / \mathrm{dL}(\%)$ & $24(39.3)$ & $18(40.9)$ & $6(35.3)$ & $1.3(0.4-4.0)$ \\
\hline Peripheral oxygen saturation $<93 \%(\%)$ & $17(27.9)$ & $13(29.5)$ & $4(23.5)$ & $1.4(0.4-4.9)$ \\
\hline Arterial pO2 on admission <60 mmHg (\%) & $30(49.2)$ & $25(56.8)$ & $5(29.4)$ & $3.2(1.0-10.5)$ \\
\hline Arterial pCO2 on admission $>40$ mmHg (\%) & $13(21.3)$ & $10(22.7)$ & $3(17.6)$ & $1.4(0.3-5.7)$ \\
\hline Worst P/F during hospitalization <200 (\%) & $22(36.1)$ & $17(38.6)$ & $5(29.4)$ & $1.5(0.4-5.0)$ \\
\hline
\end{tabular}

$60 \mathrm{mmHg}$ respectively. Nonetheless, we found no association between fibrinogen levels and dyspnea. Among 34 patients with CRP above $50 \mathrm{mg} / \mathrm{L}, 21$ had $\mathrm{pO}_{2}$ lower than $60 \mathrm{mmHg}(61.8 \%)$ compared to 9 (33.3\%) patients with CRP below $50 \mathrm{mg} / \mathrm{L}$ $\left(\chi^{2} 4.87\right.$, OR $3.2,95 \%$ CI 1.1-9.3, $\left.\mathrm{p}=0.03\right)$. No association was found when the threshold was lowered to $10 \mathrm{mg} / \mathrm{L}$ or $5 \mathrm{mg} / \mathrm{L}$.

\section{DISCUSSION}

\section{AND CONCLUSIONS}

Herein we present a retrospective observational study on 25-OH-vitamin D serum levels in patients admitted to the inpatient ward for COVID-19. We found a large prevalence of vitamin $\mathrm{D}$ deficiency in our cohort, with approximately $70 \%$ of the pa-

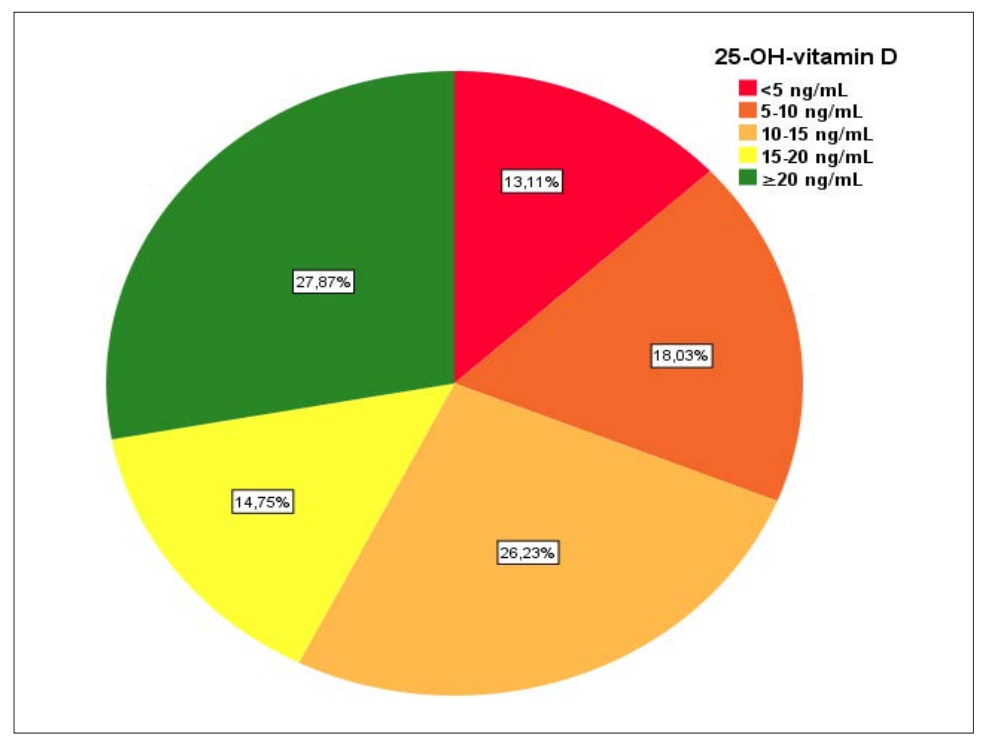

Figure 1 - Vitamin D serum levels in the study population. 


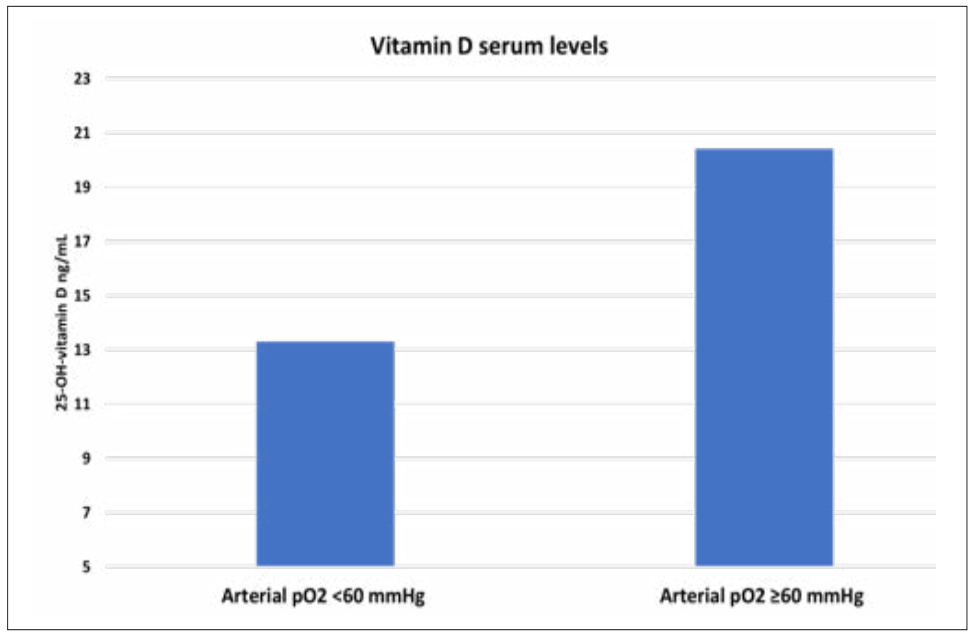

Figure 2 - Vitamin D serum levels and arterial $\mathrm{pO}_{2}$ on admission, $\mathrm{p}=0.03$.

tients with $25-\mathrm{OH}$-vitamin $\mathrm{D}<20 \mathrm{ng} / \mathrm{mL}$, a threshold that is largely recognized as representing deficiency. More than half of the subjects had less than $15 \mathrm{ng} / \mathrm{mL}$ and approximately one out of ten had less than 5 $\mathrm{ng} / \mathrm{mL}$, corresponding to severe deficiency. Patients with lower $(<60 \mathrm{mmHg})$ arterial $\mathrm{pO}_{2}$ measured at admission had significantly lower vitamin D serum levels compared to patients with higher $(\geq 60 \mathrm{mmHg})$ arterial $\mathrm{pO}_{2}$. We also found an inverse relationship between vitamin D levels and CRP levels, a well-known marker of inflammation. Of note, we did not find an association between CRP above $10 \mathrm{mg} / \mathrm{L}$ or above 5 $\mathrm{mg} / \mathrm{L}$ and arterial $\mathrm{pO}_{2}$ on admission. Therefore, we can speculate that vitamin $\mathrm{D}$ can play a role as a marker of the inflammatory response, especially in patients without a dramatic increase in CRP $(<10 \mathrm{mg} / \mathrm{L})$. In addition, in our cohort, tocilizumab was prescribed only to vitamin $\mathrm{D}$ deficient patients, possibly in relation to the worse clinical presentation. Indeed, IL-6 hyperexpression has been associated with the cytokine release syndrome in COVID-19 patients and tocilizumab, a monoclonal antibody that blocks the IL- 6 receptor, showed promising results in severely ill COVID-19 patients $(4,9)$. In line with our results, vitamin D deficiency had been reported largely prevalent in severely ill patients admitted to Intensive Care Units (ICUs) (10) and had been associated with more severe
ARDS and with worse clinical outcomes in patients admitted to $\operatorname{ICU}(11,12)$. Moreover, in patients admitted to UTIs with ARDS, cholecalciferol administration has been associated with shorter hospitalization and decreased mortality, especially among vitamin D deficient patients (13), and vitamin $\mathrm{D}$ administration has been associated with fewer respiratory infections and decreased levels of serum IL-6 (14, 15). In this scenario, vitamin D serum levels might play an important role not only in the prognosis of COVID-19 patients but also in the pathogenesis of the inflammatory response (16). It has recently been speculated, mostly based on indirect evidence, that vitamin $\mathrm{D}$ might have a role in reducing the risk of COVID-19 and experts have advised to supplement COVID-19 patients with large doses of cholecalciferol $(7,17)$. At a population level, a negative correlation between vitamin $\mathrm{D}$ and the number of COVID-19 cases was observed (18). However, to date, direct evidence linking hypovitaminosis D and COVID-19 is missing and a causative role of vitamin D deficiency on COVID-19 susceptibility is yet to be determined. Large interventional placebo-controlled studies are needed to further determine the role of vitamin $\mathrm{D}$ in COVID-19.

Our study has strengths and limitations. We have enrolled a fairly homogenous sample for the analysis, and we have collected a large set of clinical and laboratory parameters. However, this is an observational study and no definitive assumptions on the causal role of vitamin $\mathrm{D}$ deficiency on COVID-19 severity can be drawn. No control group has been included in the study and a similar vitamin D deficiency may be expected in subjects with similar baseline characteristics or critically ill patients. Nevertheless, vitamin D could be considered at least a prognostic marker of disease severity in COVID-19 patients.

In conclusion, COVID-19 patients had a great prevalence of vitamin D deficiency, which is associated with a worse clinical presentation and worse arterial $\mathrm{pO}_{2}$ at admission. Vitamin D can be a novel marker of disease severity in COVID-19 patients. 
Further interventional placebo-controlled studies of vitamin D in COVID-19 patients are urgently needed to determine its role on disease severity.

\section{Contributions}

Conceptualization, Giovanni Adami, Alessandro Giollo, Maurizio Rossini; Data curation, Giovanni Adami, Alessandro Giollo, Eugenia Bertoldo; Formal analysis, Giovanni Adami; Investigation, Giovanni Adami, Alessandro Giollo, Angelo Fassio, Camilla Benini, Eugenia Bertoldo, Francesco Bertoldo, Giovanni Orsolini, Luca Idolazzi, Ombretta Viapiana, Sandro Giannini, Giovanni Passeri, Evelina Tacconelli, Claudio Micheletto, Davide Gatti, Maurizio Rossini; Project administration, Maurizio Rossini; Supervision, Maurizio Rossini; Validation, Giovanni Adami, Maurizio Rossini; Writing - original draft, Giovanni Adami, Alessandro Giollo; Writing - review \& editing, Giovanni Adami, Alessandro Giollo, Angelo Fassio, Camilla Benini, Eugenia Bertoldo, Francesco Bertoldo, Giovanni Orsolini, Luca Idolazzi, Ombretta Viapiana, Sandro Giannini, Giovanni Passeri, Evelina Tacconelli, Claudio Micheletto, Davide Gatti, Maurizio Rossini.

\section{Conflicts of interest}

All authors have completed the ICMJE uniform disclosure form at www.icmje. org/coi_disclosure.pdf (available on request from the corresponding author) and declare no conflicts of interest.

\section{Patient and Public Involvement state- ment}

This research was done without patient involvement. Patients were not invited to comment on the study design and were not consulted in order to develop patient relevant outcomes or interpret the results. $\mathrm{Pa}$ tients were not invited to contribute to the writing or editing of this document for readability or accuracy.

\section{Ethical approval}

The institutional review board of the University of Verona approved this study (2598CESC).

\section{Data sharing}

No additional data available.

\section{Transparency declaration}

The lead author (the manuscript's guarantor) affirms that the manuscript is an honest, accurate, and transparent account of the study being reported; that no important aspects of the study have been omitted; and that any discrepancies from the study as planned (and, if relevant, registered) have been explained.

\section{REFERENCES}

1. Grasselli G, Pesenti A, Cecconi M. Critical Care Utilization for the COVID-19 Outbreak in Lombardy, Italy: Early Experience and Forecast During an Emergency Response. JAMA. 2020 [Epub ahead of print, doi: https:// doi.org/10.1001/jama.2020.4031].

2. Zhou P, Yang X-L, Wang X-G, et al. A pneumonia outbreak associated with a new coronavirus of probable bat origin. Nature. 2020; 579: 270-3.

3. Cavalli G, Luca GD, Campochiaro C, et al. Interleukin-1 blockade with high-dose anakinra in patients with COVID-19, acute respiratory distress syndrome, and hyperinflammation: a retrospective cohort study. Lancet Rheumatol. 2020 [Epub ahead of print, doi: https://doi. org/10.1016/S2665-9913(20)30127-2].

4. Toniati P, Piva S, Cattalini M, et al. Tocilizum$\mathrm{ab}$ for the treatment of severe COVID-19 pneumonia with hyperinflammatory syndrome and acute respiratory failure: A single center study of 100 patients in Brescia, Italy. Autoimmun Rev. 2020; 102568. 2020 [Epub ahead of print, doi: https://doi.org/10.1016/j.autrev. 2020.102568].

5. Prietl B, Treiber G, Pieber TR, Amrein K. Vitamin D and immune function. Nutrients. 2013; 5: 2502-21.

6. Cashman KD, Dowling KG, Škrabáková Z, et al. Vitamin D deficiency in Europe: pandemic? Am J Clin Nutr. 2016; 103: 1033-44.

7. Grant WB, Lahore H, McDonnell SL, et al. Evidence that vitamin D supplementation could reduce risk of influenza and COVID-19 infections and deaths. Nutrients. 2020; 12 [Epub ahead of print, doi: https://doi.org/10.3390/nu12040988].

8. Lange NE, Litonjua A, Hawrylowicz CM, Weiss S. Vitamin D, the immune system and asthma. Expert Rev Clin Immunol. 2009; 5: 693-702.

9. Luo P, Liu Y, Qiu L, et al. Tocilizumab treatment in COVID-19: A single center experience. J Med Virol. 2020 [Epub ahead of print, doi: https://doi.org/10.1002/jmv.25801]. 
10. Zajic P, Amrein K. Vitamin D deficiency in the ICU: a systematic review. Minerva Endocrinol. 2014; 39: 275-87.

11. Putzu A, Belletti A, Cassina T, et al. Vitamin D and outcomes in adult critically ill patients. A systematic review and meta-analysis of randomized trials. J Crit Care. 2017; 38: 109-14.

12. Dancer RCA, Parekh D, Lax S, et al. Vitamin $\mathrm{D}$ deficiency contributes directly to the acute respiratory distress syndrome (ARDS). Thorax. 2015; 70: 617-24.

13. Amrein K, Schnedl C, Holl A, et al. Effect of high-dose vitamin D3 on hospital length of stay in critically ill patients with vitamin D deficiency: the VITdAL-ICU randomized clinical trial. JAMA. 2014; 312: 1520-30.

14. Berry DJ, Hesketh K, Power C, Hyppönen E. Vitamin D status has a linear association with seasonal infections and lung function in British adults. Br J Nutr. 2011; 106: 1433-40.
15. Miroliaee AE, Salamzadeh J, Shokouhi S, Sahraei Z. The study of vitamin D administration effect on CRP and Interleukin-6 as prognostic biomarkers of ventilator associated pneumonia. J Crit Care. 2018; 44: 300-5.

16. Chabrol T, Wion D. Randomized clinical trials of oral vitamin D supplementation in need of a paradigm change: The vitamin $\mathrm{D}$ autacoid paradigm. Med Hypoth. 2020; 134: 109417.

17. Chakhtoura M, Napoli N, Fuleihan GEH. Myths and facts on vitamin D amidst the COVID-19 pandemic. Metab Clin Exp. 2020; 154276 [Epub ahead of print, doi: https://doi. org/10.1016/j.metabol.2020.154276].

18. Ilie PC, Stefanescu S, Smith L. The role of vitamin $\mathrm{D}$ in the prevention of coronavirus disease 2019 infection and mortality. Aging Clin Exp Res. 2020 [Epub ahead of print, doi: https://doi.org/10.1007/s40520-020-01570-8]. 